\title{
Author Index to Volume 26
}

Agata $\mathrm{Y}, 336$

Alpan $G, 570$

Altenburger KM, 135

Anday EK, 576

Ank BJ, 135

Anthony BF, 383

Arab N, 58

Arner P, 255

Axelsson IEM, 614

Bachmann C, 260

Baldzer K, 188

Baron K, 548

Barrish J, 500

Barshop B, 140

Baumgartner ER, 260

Baxter RC, 308

Baylen BG, 336

Beitins IZ, 320

Belik J, 548

Bell EF, 121

Benson DW, Jr, 438

Berger HM, 20

Berkowitz ID, 558

Berner M, 200

Berry SA, 410

Bhat R, 565

Bhutani VK, 434

Biancani P, 39

Bisaillon S, 290

Blauenstein U, 260

Bolas NM, 356

Bolme P, 255

Bossi E, 478

Boudreau N, 330

Boyd RDH, 109

Bradley LM, 175

Breuer J, 536

Brogan M, 188

Brusilow SW, 77

Buescher ES. 43

Büller HA, 232

Cairo MS, 395

Carlo WA, 583

Carrick BM, 3

Carrigan TA, 188

Cella SG, 316

Chang KJ, 34

Chan HSL, 154

Chan JCM, 204

Chantarojanasiri T, 558

Chessex P, 290

Childs B, 390

Christensen MA, 208

Christensen RD, 531

Christiansen JL, 11

Chung KJ, 536

Ciurea D, 88

Clark EB, 438

Clausen N, 158

Clements JF, 491

Clyman RL, 570

Coceani F, 330

Cohen ME, 576

Concepcion NF, 383

Corn PG, 54

Crissinger KD, 102

Crouch LS, 400

Curzi-Dascalova L. 347
Czaja JF, 175

Dahlin I, 106

D'Allest AM, 347

Daumit $G, 576$

Dean JM, 558

DeGiulio PA, 593

Delivoria-Papadopoulos M, 593

Deoras KS, 434

Descos BP, 410

Desjeux J-F, 237

DiFiore JM, 583

DiMaio M, 88

Drakenberg K, 128

Dykes FD, 188

Edwards BB, 250

Eeckhout Y, 125

Ebrenkranz RA, 298

Eklöf AC, 128

Emmanouilides GC, 336

Emrie PA, 167

Erve JC, 250

Fahey JT, 180

Felipe A, 448

Feltes TF, 94

Fisher JH, 167

Fliesen T, 415

Fomon SJ, 250

Foreman JW, 204

Foster CM, 320

Frank L, 16

Frenck RW, Jr., 43

Friedli B, 200

Friedman WF, 98

Gardiner EE, 639

Gardiner RM, 356

Garst M, 140

Gauda EB, 583

Gaultier $\mathrm{Cl}, 347$

Georgieff MK, 467

Gerard $G, 415$

Gerbaut L, 351

Gershwin ME, 470

Gettner PA, 298

Ghishan F, 58

Gibson RL, 241

Giddens DP, 188

Gil J, 88

Gillis CN, 420

Gillman MJ, 362

Girardin E, 200

Goldblatt PJ, 63

Goldstein RE, 175

Golub MS, 470

Gordon JB, 325

Gotteland M, 237

Grand RJ, 232

Granger DN, 102

Granoff DM, 491

Gross CR, 410

Gross SJ, 598

Gruber H, 140

Guilleminault $\mathrm{Ch}, 347$

Gunning WT. 63

Guth BD, 536

Guy DG, 250

\author{
Haas RH, 536 \\ Haddad GG, 34 \\ Hahn E, 154 \\ Hakim TS, 325 \\ Halbert K, 633 \\ Hale PM, 320 \\ Hall K, 128 \\ Hanai J, 603 \\ Hansen TN, 94, 425 \\ Haramati A, 54 \\ Hargrave BY, I \\ Harper RM, 343 \\ Hashimoto T, 145, 150 \\ Hassing JM, 320 \\ Haubart RE, 482 \\ Heiman HS, 629 \\ Henderson WR, 241 \\ Hendrickx AG, 470 \\ Herschkowitz N, 478 \\ Hertzberg T, 106 \\ Hesselink JR, 536 \\ Heyman M, 237 \\ Heymann MA, 83 \\ Hillemeier C, 39 \\ Hintz RL, 308 \\ Hitzig WH, 377 \\ Hoefler G, 67 \\ Hoefler S, 67 \\ Hoffman HS, 576 \\ Holzknecht RA, 77 \\ Hoppel CL, 63 \\ Hopwood NJ, 320 \\ Hortop J, 325 \\ Hostetter MK, 116 \\ Hou Q, 554 \\ Hughes SF, 438 \\ $\mathrm{Hu}$ N, 438 \\ Hutchens TW, 618,623 \\ Hyman PE, 458
}

Ikegami M, 336

Irestedt L, 106

Isolauri E, 237

Ivarsson SA, 614

Ives NK, 356

Iwai $Y, 49$

Iwamoto HS, 1

Janghorbani M, 250, 298

Jarrie! S, 425

Jobe AH, 336

Johnston RB, Jri, 135

Jones SA, 188

Joyner RW, 543

Käăr M-L, 404

Kainer G, 204

Kalenga M, 125

Kaneda M, 246

Kaplan SL, 500

Karpe B, 255

Kattan M, 88

Kawaguchi Y, 246

Kawai T, 603

Keen CL, 470

Kelch RP, 320

Keng J, 639

Kennedy KA, 400

Keteslegers J-M, 415

Kim KM, 49
Kim SY, 588

Kimura S, 458

Kirby ML, 11

Kishi T, 246

Klitzner $\Upsilon$ S, 98

Kloepper RF, 482

Kluge KA, 343

Knip M, 404

Kobayashi M, 246

Ko D, 204

Koehler RC, 558

Kohler E, 478

Komazawa Y, 246

Kugo M, 227

Kurczynski TW, 63

Lafeber HN, 294

Lagercrantz H, 106

Lähteenmāki A, 25

Langer $R, 452$

Lautala P, 404

Lee PDK, 308

Lemmons RM, 73

Lepage G, 290

Leppäluoto J, 404

Leslie J, 140

Light RB, 548

Lindeman JHN, 20

Lisowski KM, 73

Lister G, 180

Loche $\$, 316$

Lönnerdal B, 470

Maes M, 415

Maeta $\mathrm{H}, 565$

Magnuson JS, 618, 623

Maiter D, 415

Marcus C, 255

Martin R, 583

Maruko T, 246

Mason EO, Jr, 500

Matalon R, 140

Mauray F, 570

Mauss D, 395

Mayock E, 196

Mayumi M, 49

McDougall JG, 6

Mela-Riker L, 220

Mendes T, 320

Michael JR, 558

Michals K, 140

Miettinen PJ, 25

Mikawa $\mathrm{H}, 49$

Miller MJ, 583

Mills MM, 467

Mishra OP, 593

Miyake $\mathrm{H}, 603$

Momma K, 442

Montgomery RK, 232

Moriette G, 351

Moser A, 67

Moser H, 67

Mughal MZ, 109

Müller EE, 316

Mullon CJ-P, 452

Mulroney SE, 54

Mulroy MJ, 11

Murdaugh E, 215

Murphy TV, 491

Nadal D, 377 
Nagasawa $\mathrm{H}, 145$

Nakamura H, 227

Nakano M, 204

Nanbu M, 49

Nedelcoux H, 347

Nelli CM, 298

Nelson SE, 250

Newberry RC, 639

Nielsen HC, 16

Nielsen JH, 158

Nishi M, 603

Nowakowski RW, 462

Nyhan WL, 140

O'Donovan BH, 121

Okada K, 246

Olin KL, 470

Oliphant M, 598

Oliver TK, Jr, 287

Oltey PM, 330

Orii T, 145,150

Osaka T, 543

Otonkoski T, 25

Padbury JF, 336

Paltauf F, 67

Panula $P, 25$

Pascbke E, 67

Pastor-Anglada M, 448

Patrick CC, 500

Patrick GS, 500

Paunier L, 200

Pencharz PB, 154

Peng H-J, 486

Penn RB, 429

Perheentupa J, 25

Persson B, 128

Pescovitz $\mathrm{OH}, 410$

Pesonen E, 536

Philipps A, 128

Pineault M, 290

Pinheiro JMB, 420

Pintor C. 316

Pisoni RL, 73

Pitt BR, 420

Pizer LI, 135

Plaeger-Marshall S, 135

Poorthuis BJHM, 20

Powell DR, 308

Praud JP, 347

Probst A, 260
Puggioni R, 316

Rabinovitch M, 330

Räihä NCR, 6l4

Raju TNK, 565, 588

Ramza BM, 543

Rantala H, 404

Redding GJ, 241

Relier JP, 351

Remesar X, 448

Richardson P, 425

Rings EHHM, 232

Ripalda MJ, 366

Rivest RW, 200

Robinson NR, 109

Rogers MC, 558

Rogers RR, 250

Roscher AA, 67

Rosenberg AA, 215

Rosen CL, 34

Ross R, 633

Roth RA, 593

Rouge JC, 200

Roy CC, 290

Rubens CE, 241

Rudolph AM, I

Rudolph N, 366

\section{Sano K, 227}

Sara V, 128

Satoh T, 246

Sato Y, 603

Sauer PJJ, 294

Saugier P, 351

Schechtman VL, 343

Schellhase DE, 167

Schleien CL, 558

Schreiber MD, 83

Schrijver J, 20

Schutgens RBH, 145

Scriver CR, 388

Seegmiller JE, 140

Sender L, 395

Shaffer TH, 429, 434

Shannon JM, 167

Sharp HL, 410

Sherman WR, 482

Sherwonit EA, 298

Shimada S, 565

Shimozawa N, 150

Sibley CP, 109
Singh $M, 34$

Sjögren B, 128

Slagle TA, 598

Slotkin TA, 554

Snape WJ, Jr, 458

Soifer SJ, 83

Sollevi A, 106

Sonnenfeld T, 255

Sosenko IRS, 16

Speek AJ, 20

Sperry JB, 308

Stabilini L, 316

Stadt HA, II

Stallings VA, 154

Stambouly JJ, 175

Standaert TA, 196

Stein DT, 208

Stevenson DK, 362

Stiehm ER, 135

Stonestreet BS, 467

Strobel S, 486

Sulkers EJ, 294

Suormala TM, 260

Suzuki X, 150

Sweetman L, 145

Sybicki MA, 232

Takao A, 442

Takasugi N, 603

Takeda T, 603

Tanaka M, 49

Tanaka $Y, 246$

Tanihiro M, 246

Tan RC, 543

Tapanainen P, 404

Taverna $\mathrm{Ch}, 377$

Taylor KB, 462

Thoene JG, 73

Thompson JN, 462

Thurston $\mathrm{JH}, 482$

Ting BTG, 298

Tome D, 237

Tomomasa T, 458

Tordet $C, 351$

Tosone CM, 452

Towstoless MK, 6

Toy C, 395

Traystman RJ, 558

Truog WE, 241

Tsai MY, 77

Tsang RC, 633
Tuchman M, 77

Turner MW, 486

Twiggs GA, 196

Ueda K, 246

Uetani $Y, 227$

Underwood LE, 415

Uriu-Hare JY, 470

Vadhan-Raj S, 43

Vaisman $N, 154$

van de Ven C, 395

Vannucci RC, 208

van Zoeren-Grobben D, 20

Vella $G, 330$

Vest M, 260

Vidyasagar D, 565

Vitulli WF, 496

Vlessis AA, 220

Vreman HJ, 362

Wagerle LC, 593

Wallin $R, 370$

Walterspiel JN, 496

Walti $\mathrm{H}, 351$

Warshaw JB, 400

Wassner SJ, 608

Watchko JF, 196

Weaver LT, 31

Weisdorf SA, 410

Weisman LE, 629

Weitzman SS, 154

White CW, 215

Wick H, 260

Widness JA, 467

Williams JE, 298

Winter S, 140

Wintour EM, 6

Wolff JA, 140

Wolfson MR, 429, 434

Wong SL, 366

Woodrum DE, 196

Yagi H, 458

Yamaguchi S, 145

Yip T, 618,623

Yodoi J, 49

Y'umiba C, 246

Zach TL, 116

Ziegler EE, 250 